\title{
Magnetoquantum oscillations in the resistance of metallic point contacts: Influence of nonequilibrium phonons
}

\author{
N. L. Bobrov ${ }^{1,2, *}$, J. A. Kokkedee ${ }^{1}$, N. N. Gribov ${ }^{1,2}$, I. K. Yanson², A. G. M. Jansen ${ }^{1}$, P. Wyder ${ }^{1}$ \\ ${ }^{1}$ High Magnetic Field Laboratory, \\ Max-Planck Institut fur Festkorperforschung and Centre National de la Recherche Scientifique, \\ F-38042, Grenoble, Cedex 09, France \\ ${ }^{2}$ B.I. Verkin Institute for Low Temperature Physics and Engineering, \\ of the National Academy of Sciences of Ukraine, \\ prospekt Lenina, 47, Kharkov 61103, Ukraine \\ E-mail address: bobrov@ilt.kharkov.ua
}

(Dated: August 28, 2018; Published Physica B 218, 42(1996))

\begin{abstract}
The amplitude of magnetoresistance quantum oscillations of $A l$ and $B e$ point contacts in a magnetic field parallel to the contact axis has been studied as a function of voltage applied over the contact. It was found that for one group of contacts the oscillation amplitude nonmonotonously increases with the bias voltage increase, while for the other part of the contacts a decrease of amplitude was observed. The scattering of electrons with nonequilibrium phonons and also phonon-phonon collisions will be discussed as the possible reasons of the observed effects.

PACS numbers: 71.38.-k, 73.40.Jn, 74.25.Kc, 74.45.+c
\end{abstract}

\section{INTRODUCTION}

The resistance of metallic point contacts at low temperatures shows the oscillations as a function of magnetic field, which are due to the Landau quantization of the conduction electron energy spectrum [1, 2]. An increase in temperature reduces the amplitude of oscillations. The corresponding reduction factor is

$$
R_{T}=\frac{2 \pi^{2} n k_{B} T / \hbar \omega_{c}}{\operatorname{sh}\left(2 \pi^{2} n k_{B} T / \hbar \omega_{c}\right)} ;
$$

where $n$ is the harmonic number in the oscillations and $\omega_{c}=e B / m^{*} c$ the cyclotron frequency. Elastic scattering of the electrons by impurities also causes the decrease of the oscillation amplitude nearly the same as resulted from the temperature increase from actual value $T$ up to $T_{\text {eff }}=T+x$. The reduction factor (the Dingle factor) has the form

$$
R_{D}=\exp \left(-2 \pi^{2} k_{B} n x / \hbar \omega_{c}\right),
$$

where $x=\hbar / 2 \pi k_{B} \tau$ is the Dingle temperature, and $\tau$ is the elastic lifetime of the electrons. As follows from Eqs.

(1) and (2) the ratio between the first and the second harmonic of the oscillations is

$$
A_{1} / A_{2}=C \operatorname{ch}\left(2 \pi^{2} k_{B} / \hbar \omega_{c}\right) \exp \left(\pi / \omega_{c} \tau\right),
$$

\footnotetext{
${ }^{*}$ Corresponding author.
}

where the constant $C$ has a meaning of the ratio of the harmonics at $T=0$ in the absence of scatterers. From the theory for noninteracting particles it follows that the Dingle temperature can be affected by any electron scattering process, including electron-phonon collisions [3]. At the same time, with many-body interactions taken into account, the scattering of electrons on phonons seems to have no effect on the Dingle temperature [3]. The study of the influence of electron-phonon collisions on the amplitude of magnetoquantum oscillations in traditional experiments is limited to metals with low Debye energy, in which phonons can be excited at a tem, perature low enough to observe the effect. Metallic point contacts present a unique opportunity to study the influence of nonequilibrium phonons generated in the contact on the quantum oscillations. Just by applying the bias over the contact, the nonequilibrium phonons can be generated in the constriction with any possible energy up to $\mathrm{eV}$ [4]. In this work we study the effect of electron- phonon scattering on the amplitude of the resistance oscillations of $A l$ and $B e$ point contacts in a magnetic field parallel to the contact axis.

\section{EXPERIMENTAL DETAILS}

Point contacts were formed between edges of two single crystal electrodes with the same crystallographic orientations. The applied magnetic field was always parallel 


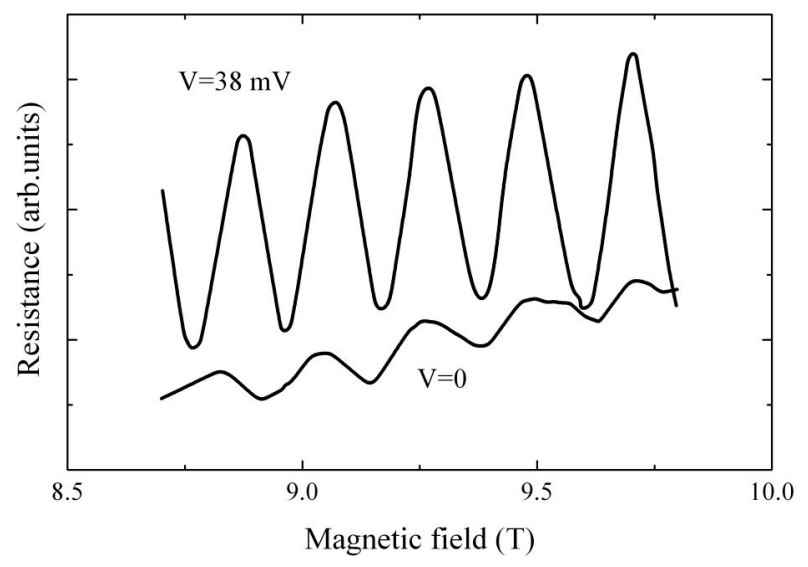

FIG. 1: Fragments of magnetoresistance oscillations of an $\mathrm{Al}$ point contact of $R_{0}=1.41 \Omega$ for two different bias voltages.

to the contact axis. All the experiments were carried out at the temperature $1.3 \mathrm{~K}$. The first and the second derivatives of the $I-V$ curve were measured by standard modulation technique. To determine the energy dependence of the amplitude of magnetoresistance oscillations the following procedure was used. For several fixed bias voltage $d V / d I(B)$ dependencies were recorded in the same interval of magnetic field (usually in between 8 and $10 T)$. Fig. 1 shows the fragments of $d V / d I(B)$ curves for an $A l$ point contact for two bias voltages. The amplitude of oscillations $A_{1}$ and its second harmonic $A_{2}$ was obtained by means of Fourier analysis. For one group of contacts the voltage dependence $A_{1}(\mathrm{eV})$ was found to vary nonmonotonously with applied voltage with similarities to the point contact spectrum of electron-phonon interaction. Such an effect was observed for low Ohmic $A l$ point contacts (Fig. 2(a)) and Be contacts (Fig. 3(a)).

High Ohmic $A l$ point contacts show a decrease of the amplitude of oscillations in their magnetoresistance with the bias voltage increase (Fig. 4(a)).

\section{DISCUSSION}

Phonons generated in point contact are the result of the energy relaxation of electrons injected from the contact. In analogy with elastic impurity scattering, the scattering of electrons on these nonequilibrium phonons will influence the amplitude of magnetoquantum oscillations. As with the elastic scattering [5], phonon-induced scattering within the constriction region will cause the randomization of the momenta of electrons. This randomization will lead to an effective increase of the $z$ -

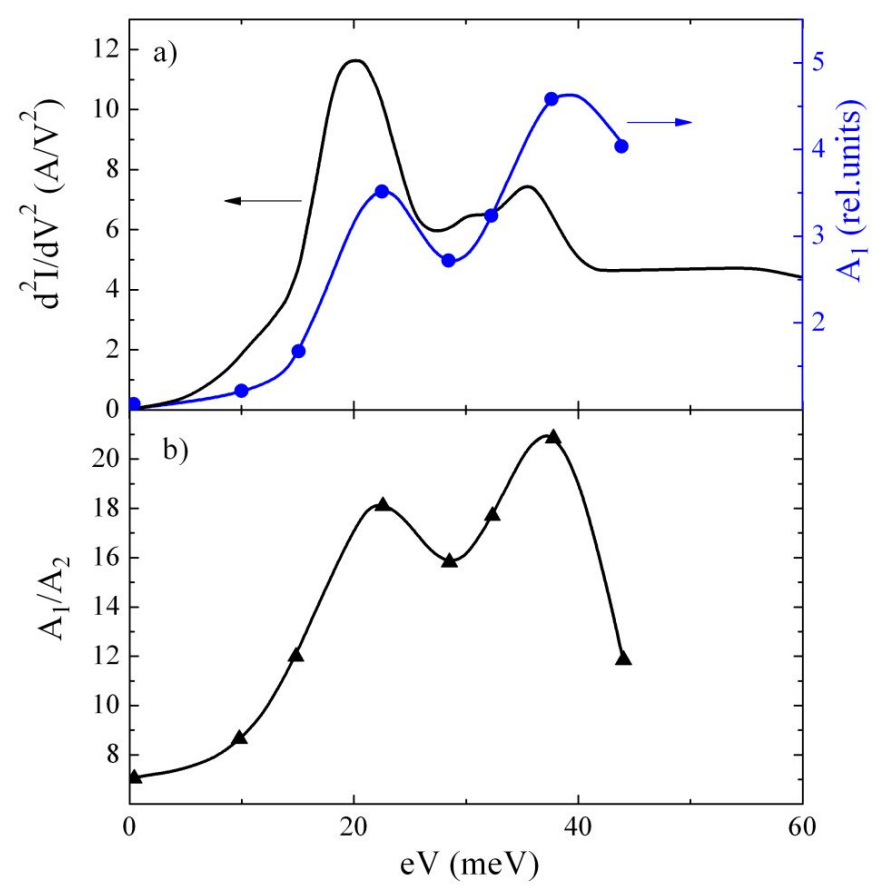

FIG. 2: (a) Point contact spectrum of an $A l$ point contact of $R_{0}=1.41 \Omega$ and relative magnitude of the first harmonic of the resistance oscillations versus bias voltage; $\Delta R / R_{0} \approx 5 \times 10^{-4}$ at $V=0$ and $B=9.5 T$.

(b) Ratio between the amplitude of the first and the second harmonic of the magnetoresistance oscillations versus bias voltage.

component of the transport velocity of electrons from the extremal cross section of the Fermi surface and correspondingly to an increase of quantum oscillations in the point contact magnetoresistance. At the same time, the scattering of electrons on nonequilibrium phonons in the boundaries of the point contact will cause the increase of the Dingle temperature and thus a decrease of the oscillation amplitude. The resulting effect will be determined by the balance between these two processes.

It is obvious that the purity of the metal in the region adjacent to the constriction area is an important factor affecting the oscillations of the electron density of states. As follows from Eq. (2) the oscillation amplitude has nonlinear dependence on the concentration of the impurity scatterers. In the case of a large value of $\omega_{c} \tau$ (low impurity concentration) it saturates and exponentially drops at small values of $\omega_{c} \tau$. Hence, if the concentration of defects in the point contact boundaries is low, the small increase in the number of the electronphonon scattering events leads to insignificant decrease of the oscillation amplitude. However, if the impurity 


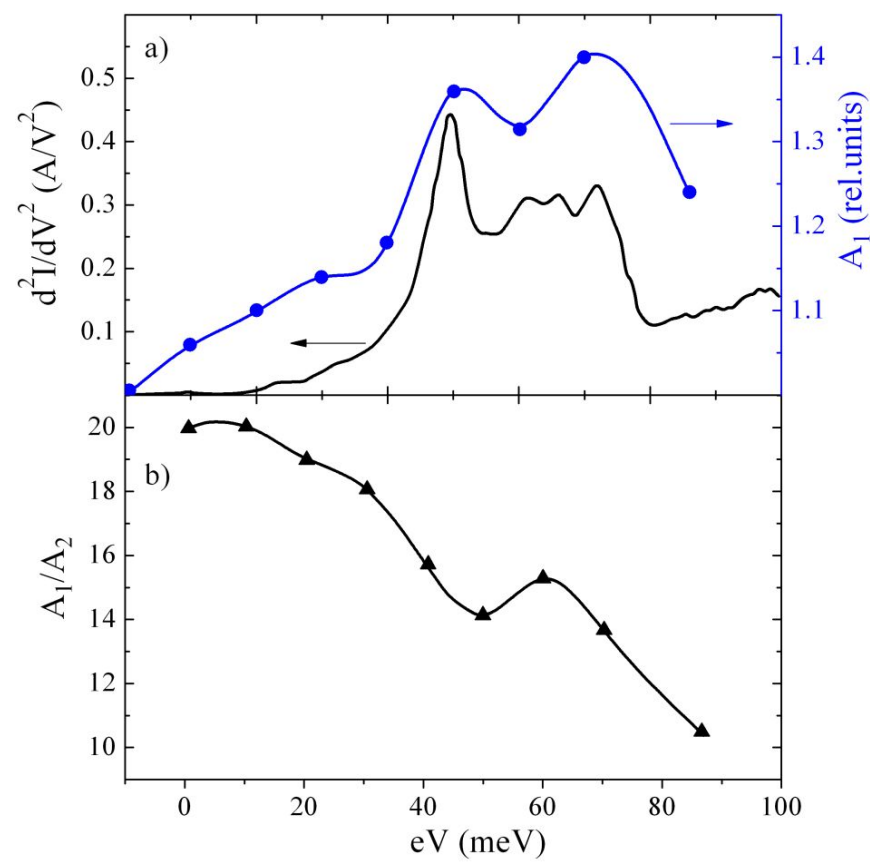

FIG. 3: (a) Point contact spectrum of an Be point contact of $R_{0}=8.66 \Omega$ and relative magnitude of the first harmonic of the resistance oscillations versus bias voltage; $\Delta R / R_{0} \approx 2 \times 10^{-3}$ at $V=0$ and $B=9.5 T$.

(b) Ratio between the amplitude of the first and the second harmonic of the magnetoresistance oscillations versus bias voltage.

concentration in the boundaries is high enough, a similar increase in the number of electron-phonon collisions may dramatically affect the oscillations.

Taking into account all the factors mentioned above one can expect an increase of the amplitude of magnetoresistance oscillations in relatively large (compare to the diffusive mean free path for phonons) low Ohmic contacts with pure boundaries. Nonequilibrium phonons generated in such contacts are accumulated within the constriction area and play an effective part in the isotropization of the electron momenta. Purity of the boundaries is of great importance because the oscillations are mainly the result of quantizing conditions there. Fig. 2(a) shows the example of $A_{1}(\mathrm{eV})$ dependence for one of low Ohmic $A l$ point contact. The oscillation amplitude dramatically increases with the bias voltage increase. A low value of the ratio $A_{1} / A_{2}$ at $e V=0$ (Fig. 2(b)) indicates the high purity of the boundaries of this contact. In the case of small (high Ohmic) contacts the generated phonons leave the constriction area. If, additionally, the boundaries of the contact are not pure enough (high value of $\left.A_{1} / A_{2}\right)$, the decrease of the amplitude of magnetore-

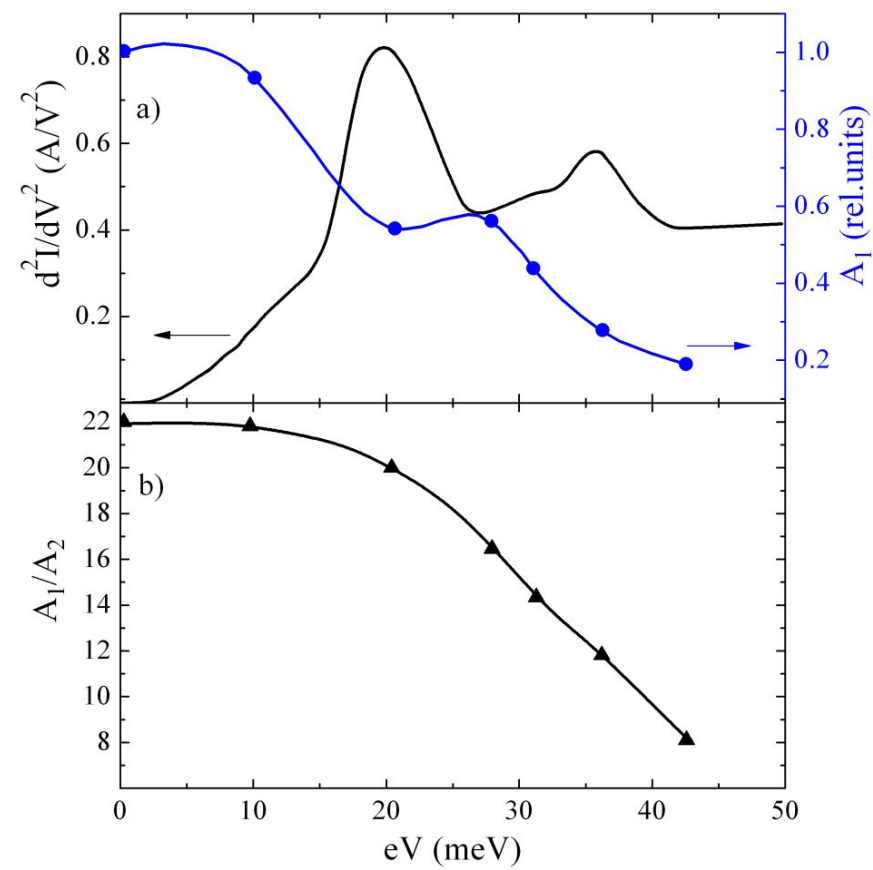

FIG. 4: (a) Point contact spectrum of an $A l$ point contact of $R_{0}=8.73 \Omega$ and relative magnitude of the first harmonic of the resistance oscillations versus bias voltage; $\Delta R / R_{0} \approx 3 \times 10^{-3}$ at $V=0$ and $B=9.5 T$.

(b) Ratio between the amplitude of the first and the second harmonic of the magnetoresistance oscillations versus bias voltage.

sistance oscillations is observed (see Fig. 4).

As for $B e$, the increase of the oscillation amplitude with bias rise was observed for all contacts (Fig. 3). This is probably due to high rigidity of the $B e$ crystal lattice, which prevents the spreading of the deformation to the boundaries during the contact formation. These deformations are concentrated near the surface, forming the effective reflectors, which return the phonons back to the contact.

The given model can not only explain the monotonous part of the voltage dependence but also its similarity to the electron phonon interaction spectrum. The group velocity of the phonons generated by the electron flow depends on their energy and is minimum for phonons with energies close to the maxima of the phonon density of states. This means that the phonon energy relaxation length will decrease stepwise at voltages corresponding to the characteristic phonon frequencies. Therefore, one should expect the similarity of $A_{1}(e V)$ with the first derivative $d V / d I(\mathrm{eV})$ of the $I-V$ curve, which is not the case in our experiments. However, in these speculations the role of phonon-phonon collisions was not taken 
into account.

The stepwise decrease of the phonon energy relaxation length and correspondingly their group velocity at voltages higher than or of the order of typical phonon energies leads to a significant increase of the frequency of phononphonon collisions. This will result in the thermalization of phonons, which may have the following consequences:

1. Phonon-phonon collisions will decrease the number of high-frequency phonons, which are the most efficient electron scatterers because of their small group velocities, while the thermalized phonons will leave the contact area.

2. An increase of the total number of phonons in the contact boundaries, resulting from thermalization leads to a stronger suppression of oscillations in this area.

Hence, one can expect that for bias voltages higher than or of the order of characteristic phonon energies, the rise of $A_{1}(\mathrm{eV})$ will slow down or even a decrease may be observed.

Now consider the bias dependence of the ratio between the first and the second harmonics of the oscillations $A_{1} / A_{2}(e V)$. From Eq. (3) it can be readily seen that the magnitude of $A_{1} / A_{2}$ depends on the value of the electron mean free path in the region of metal where the quantization of electron density of states takes place. Due to geometry of experiments (magnetic field direction is parallel to the contact axis) the ratio $A_{1} / A_{2}$ at $e V=0$ contains the information about electron mean free path in the region adjacent to the constriction area. As for the energy dependence of $A_{1} / A_{2}$ we assume the existence of two mechanisms, which can be responsible for the behaviour observed:

1. A decrease of (quasi-)elastic mean free path of electrons in the contact boundaries due to the scattering of electrons on nonequilibrium phonons.

2. Redistribution of the relative contribution to the information about electron mean free path from the areas with different concentrations of impurities. If the impurity concentration is homogeneous over the entire contact region, the ratio $A_{1} / A_{2}$ will increase with the bias rise. If, however, the concentration of impurities decreases with the distance to the contact centre, the opposite effect will be observed.

Certainly, coexistence of both mechanisms is possible. It is obvious that the first of them will lead to an increase of the $A_{1} / A_{2}$ ratio only (see Eq. (2)).

In summary, we have studied the energy dependence of the oscillation amplitude in magnetoresistance of metallic point contacts in a magnetic field parallel to the contact axis. It was found for the first time that this dependence shows a nonmonotonous behaviour and in some cases its shape is similar to the point contact spectrum of electron phonon interaction. The additional scattering of quantized electrons on the phonons generated by the flow of electrons through the contact may be the possible reason of the observed effect.
[1] N. N. Gribov, O. I. Shklyarevskii, E. I. Ass, and V. V. Andrievskii, Fiz. Nizk. Temp. 13, 642 (1987) [Sov. J. Low Temp. Phys. 13, 363 (1987)].

[2] H. M. Swartjes, A. P. van Gelder, A. G. M. Jansen, and P. Wyder, Phys. Rev. B 39, 3086 (1989).

[3] D. Shoenberg, Magnetic Oscillations in Metals, Cambridge, Univ. Press, Cambridge (1984).
[4] I. O. Kulik, A. N. Omel'yanchuk, and R. I. Shekhter, Fiz. Nizk. Temp. 3, 1543 (1977) [Sov. J. Low Temp. Phys. 3, 740 (1977)].

[5] E. M. Bogachek and R. I. Shekhter, Fiz. Nizk. Temp. 14, 810 (1988) [Sov. J. Low Temp. Phys. 14, 445 (1988)]. 